\title{
Technical Note: Analytical Inversion of the Parametric Budyko Equations
}

\author{
Nathan G. F. Reaver ${ }^{1,2}$, David A. Kaplan ${ }^{2}$, Harald Klammler ${ }^{2,3}$, and James W. Jawitz ${ }^{4}$ \\ ${ }^{1}$ Water Institute, University of Florida, Gainesville, Florida, USA. \\ $5 \quad{ }^{2}$ Engineering School of Sustainable Infrastructure and Environment (ESSIE), University of Florida, Gainesville, Florida, USA. \\ ${ }^{3}$ Department of Geosciences, Federal University of Bahia, Salvador, Bahia, Brazil. \\ ${ }^{4}$ Soil and Water Science Department, University of Florida, Gainesville, Florida, USA.
}

Correspondence to: Nathan G. F. Reaver (nreaver@ufl.edu)

Abstract. The non-parametric Budyko framework provides empirical relationships between a catchment's long-term mean evapotranspiration $(\bar{E})$ and the aridity index, defined as the ratio of mean rainfall depth $(\bar{P})$ to mean potential evapotranspiration $\left(\overline{E_{0}}\right)$. The parametric Budyko equations attempt to generalize this framework by introducing a catchment-specific parameter $(n$ or $w$ ), intended to represent differences in catchment climate and landscape features. Many studies have developed complex regression relationships for the catchment-specific parameter in terms of biophysical features, all of which use known values of $\bar{P}, \overline{E_{0}}$, and $\bar{E}$ to numerically invert the parametric Budyko equations to obtain values of $n$ or $w$. In this study, we analytically

15 invert both forms of the parametric Budyko equations, producing expressions for $n$ and $w$ only in terms of $\bar{P}, \overline{E_{0}}$, and $\bar{E}$. These expressions allow for $n$ and $w$ to be explicitly expressed in terms of biophysical features through the dependence of $\bar{P}, \overline{E_{0}}$, and $\bar{E}$ on those same features.

\section{Introduction}

The non-parametric Budyko framework was developed to explain and describe the distinctive clustering pattern observed for the long-term average evaporative behavior across multiple catchments. This pattern emerges when the evaporative indices, $\frac{\bar{E}}{\bar{P}}$ (where $\bar{P}$ is the mean rainfall depth and $\bar{E}$ is the mean actual evapotranspiration depth), of multiple catchments are plotted against their corresponding aridity indices, $\frac{\overline{E_{0}}}{\bar{P}}$ (where $\overline{E_{0}}$ is the mean potential evapotranspiration depth). Several empirical relationships of the form,

$\frac{\bar{E}}{\bar{P}}=f_{0}\left(\frac{\overline{E_{0}}}{\bar{P}}\right)$,

25 have been proposed to describe this pattern, including (Schreiber, 1904),

$\frac{\bar{E}}{\bar{P}}=1-e^{-\frac{\overline{E_{0}}}{\bar{P}}}$,

and (Ol'Dekop, 1911) 
$\frac{\bar{E}}{\bar{P}}=\frac{\overline{E_{0}}}{\bar{P}} \tanh \left(\frac{\bar{P}}{\overline{E_{0}}}\right)$

Equations (2) and (3) were selected since they closely match the central tendency of $\frac{\bar{E}}{\bar{P}}$ across $\frac{\overline{E_{0}}}{\bar{P}}$, obey the laws of conservation 30 of energy and mass for all values of $\frac{\overline{E_{0}}}{\bar{P}}$, and approach energy limitation (i.e., $\frac{\bar{E}}{\bar{P}} \rightarrow \frac{\overline{E_{0}}}{\bar{P}}$ ) and water limitation (i.e., $\frac{\bar{E}}{\bar{P}} \rightarrow 1$ ) in the humid (i.e., $\frac{\overline{E_{0}}}{\bar{P}} \rightarrow 0$ ) and arid (i.e., $\frac{\overline{E_{0}}}{\bar{P}} \rightarrow \infty$ ) limits, respectively. The geometric mean of Eq. (2) and (3) has been shown to predict $\frac{\bar{E}}{\bar{P}}$ with $\sim 10 \%$ uncertainty (Budyko and Zubenok, 1961; Gentine et al., 2012) for ungauged basins if $\bar{P}$ and $\overline{E_{0}}$ are known.

In an attempt to generalize the non-parametric Budyko framework and explain deviations in $\frac{\bar{E}}{\bar{P}}$ from the central tendency of the empirically observed catchment clustering pattern, two parametric Budyko equations have been proposed (Turc, 1953; Choudhury, 1999; Mezentsev, 1955; Yang et al., 2008),

$\frac{\bar{E}}{\bar{P}}=\frac{\frac{\overline{E_{0}}}{\bar{P}}}{\left[1+\left(\frac{\overline{E_{0}}}{\bar{P}}\right)^{n}\right]^{\frac{1}{n}}}$,

and (Tixeront, 1964; Berkaloff and Tixeront, 1958; Fu, 1981; Zhang et al., 2004),

$\frac{\bar{E}}{\bar{P}}=1+\frac{\overline{E_{0}}}{\bar{P}}-\left(1+\left(\frac{\overline{E_{0}}}{\bar{P}}\right)^{w}\right)^{\frac{1}{w}}$

where $n$ and $w$ are dubbed "catchment-specific parameters". Equations (4) and (5) can alternatively and equivalently be

40 expressed in terms of the R-Index, $\frac{\bar{E}}{\overline{E_{0}}}$ (Yao, 1974), and humidity index, $\frac{\bar{P}}{\overline{E_{0}}}$ (Hulme et al., 1992), giving,

$\frac{\bar{E}}{\overline{E_{0}}}=\frac{\frac{\bar{P}}{\overline{E_{0}}}}{\left[1+\left(\frac{\bar{P}}{\bar{E}_{0}}\right)^{n}\right]^{\frac{1}{n}}}$,

and

$\frac{\bar{E}}{\overline{E_{0}}}=1+\frac{\bar{P}}{\overline{E_{0}}}-\left(1+\left(\frac{\bar{P}}{\overline{E_{0}}}\right)^{w}\right)^{\frac{1}{w}}$

The catchment-specific parameter ( $n$ or $w$ ) has been described as an empirical, effective parameter representing the

45 influence of all catchment biophysical features, other than $\bar{P}$ and $\overline{E_{0}}$, on $\bar{E}$ (Wang et al., 2016a). Additionally, the functional forms of the parametric Budyko equations have typically been interpreted as representing the evaporative behavior of individual catchments under different aridity indices (e.g., (Roderick and Farquhar, 2011; Wang and Hejazi, 2011; Yang and Yang, 2011; Wang et al., 2016b; Zhou et al., 2016; Shen et al., 2017; Zhang et al., 2016; Milly et al., 2018)). Utilizing these interpretations, many studies have developed complex regression relationships for the catchment-specific parameter in terms 50 of various climate and landscape features (Yang et al., 2007; Donohue et al., 2012; Yang et al., 2009; Shao et al., 2012; Li et 
al., 2013; Xu et al., 2013; Cong et al., 2015; Yang et al., 2016; Zhang et al., 2018; Abatzoglou and Ficklin, 2017; Xing et al., 2018; Zhao et al., 2020; Ning et al., 2020b; Ning et al., 2020a; Li et al., 2020b; Li et al., 2020a; Zhang et al., 2019; Ning et al., 2019; Bai et al., 2019; Ning et al., 2017). In all such studies, known values of $\bar{P}, \overline{E_{0}}$, and $\bar{E}$, estimated empirically or via modelling, are used to numerically invert the parametric Budyko equations to obtain values of the catchment-specific parameter, which are then regressed against various biophysical features. The expressions obtained from such endeavours vary considerably between studies, both in their functional forms and which biophysical features are included, making it difficult to develop a consistent mechanistic understanding of the catchment-specific parameter (Reaver et al., 2020). This difficulty could be overcome by having the explicit relationship between $n$ or $w$ and $\bar{P}, \overline{E_{0}}$, and $\bar{E}$, which would allow for $n$ or $w$ to be expressed in terms of biophysical features through the dependence of $\bar{P}, \overline{E_{0}}$, and $\bar{E}$ on those same features.

In this study, we analytically invert both forms of the parametric Budyko equations. The resulting expressions give $n$ and $w$ only in terms of $\bar{P}, \overline{E_{0}}$, and $\bar{E}$, illustrating that if $n$ and $w$ depend on any biophysical features, it is due directly to the dependence of $\bar{P}, \overline{E_{0}}$, or $\bar{E}$ on those same features. Notably, there has not been an analytical derivation illustrating how $n$ and $w$ relate to biophysical features, though the importance of doing so has been noted many times (Zhang et al., 2004; Yang et al., 2008; Donohue et al., 2012; Xu et al., 2013; Greve et al., 2015; Wang et al., 2016a; Zhang et al., 2018). The expressions

65 we develop here for $n$ and $w$ satisfy this need, providing a general expression for the dependence of $n$ and $w$ on any possible biophysical features through the dependence of $\bar{P}, \overline{E_{0}}$, and $\bar{E}$ on those same features.

\section{Analytical Expressions for $n$ and $w$}

To develop explicit analytical expressions for $n$ and $w$, we start with Eq. (4) and (5). These two equations can be algebraically manipulated into,

$70 \quad\left(e^{n}\right)^{\ln \left(\frac{\overline{E_{0}}}{\bar{E}}\right)}-\left(e^{n}\right)^{\ln \left(\frac{\overline{E_{0}}}{\bar{P}}\right)}=1$,

and

$\left(e^{w}\right)^{\ln \left(\frac{\overline{E_{0}}+\bar{P}-\bar{E}}{\bar{P}}\right)}-\left(e^{w}\right)^{\ln \left(\frac{E_{0}}{\bar{P}}\right)}=1$,

respectively. Similarly, Eq. (6) and (7) can be manipulated into

$\left(e^{n}\right)^{\ln \left(\frac{\bar{P}}{\bar{E}}\right)}-\left(e^{n}\right)^{\ln \left(\frac{\bar{P}}{E_{0}}\right)}=1$,

75 and

$\left(e^{w}\right)^{\ln \left(\frac{\overline{E_{0}}+\bar{P}-\bar{E}}{\bar{E}_{0}}\right)}-\left(e^{w}\right)^{\ln \left(\frac{\bar{P}}{E_{0}}\right)}=1$,

respectively. Eq. (8), (9), (10), and (11) all have the following general form, 
$y^{C}+z y^{D}=1$,

where $C$ and $D$ are constants related to the evaporative and aridity indices, respectively, $z$ is an arbitrary complex variable, and

$80 y$ is a function of $z$. With the constraint, $C>D>0$, Eq. (12) has a solution of the form (Hochstadt, 2012), p. 81-84,

$y(z)=\frac{1}{C} \sum_{r=0}^{\infty} \frac{(-1)^{r} z^{r} \Gamma\left(\frac{1+D r}{C}\right)}{r ! \Gamma\left(\frac{1+D r}{C}+1-r\right)}$,

where $r$ is an integer index variable, and $\Gamma$ ( ) is the gamma function. Table 1 illustrates that Eq. (8) and (9) fulfill the necessary constraints (i.e. $C>D>0$ ) for arid climates (i.e. $\overline{E_{0}}>\bar{P}$ ) and Eq. (10) and (11) do so for humid climates (i.e. $\overline{E_{0}}<\bar{P}$ ), which leads to the explicit analytical expression for $n$ and $w$,

$85 n$ or $w=\ln \left[\frac{1}{\ln (G)} \sum_{r=0}^{\infty} \frac{\Gamma\left(\frac{1+\ln (H) r}{\ln (G)}\right)}{\Gamma(r+1) \Gamma\left(\frac{1+\ln (H) r}{\ln (G)}+1-r\right)}\right]$,

where $G_{n}=\left\{\frac{\overline{E_{0}}}{\bar{E}}, \overline{\bar{P}}\right\}, G_{w}=\left\{\frac{\overline{E_{0}}+\bar{P}-\bar{E}}{\bar{P}}, \frac{\overline{E_{0}}+\bar{P}-\bar{E}}{\overline{E_{0}}}\right\}$, and $H_{n, w}=\left\{\frac{\overline{E_{0}}}{\bar{P}}, \frac{\bar{P}}{\overline{E_{0}}}\right\}$; the first and second terms inside the braces apply to arid and humid climates, respectively (see also explicit forms in Eq. (A47)-(A50) of appendix). For critical point climates, where $\overline{E_{0}}=\bar{P}$, Eq. (8), (9), (10), and (11) can be solved algebraically, giving,

$n$ or $w=\frac{\ln (2)}{\ln (\Omega)}$,

90 where $\Omega_{n}=\frac{\overline{E_{0}}}{\bar{E}}=\frac{\bar{P}}{\bar{E}}$ and $\Omega_{w}=2-\frac{\bar{E}}{\bar{E}_{0}}=2-\frac{\bar{E}}{\bar{P}}$ (see also Eq. (A51) and (A52) of appendix). The detailed derivations of Eq. (14) and (15), are presented in Appendix A. 
105 Table 1: Illustration of the condition $C>D>0$ for arid climates (Eq. (8) and (9)) and for humid climates (Eq. (10) and (11)). From Left to Right: The first column gives the equation and associated climate. The second column gives mathematical constraints that must be true given an arid or humid climate. The third column gives specific mathematical constraints derived from the climatic constraints. The last column gives the condition, $C>D>0$, for each associated equation and climate, given the climatic and derived constraints.

\begin{tabular}{|c|c|c|c|c|}
\hline $\begin{array}{l}\text { Version of } \\
\text { Eq. (14) } \\
\text { and } \\
\text { Climate }\end{array}$ & $\begin{array}{l}\text { Climatic } \\
\text { Constraint }\end{array}$ & Derived Constraints & Resulting Condition, $C>D$ & $>0$ \\
\hline $\begin{array}{l}n \text { version, } \\
\quad \text { arid }\end{array}$ & $\overline{E_{0}}>\bar{P}>\bar{E}$ & $\frac{\overline{E_{0}}}{\bar{E}}>\frac{\overline{E_{0}}}{\bar{P}}>1$ & $\ln \left(\frac{\overline{E_{0}}}{\bar{E}}\right)>\ln \left(\frac{\overline{E_{0}}}{\bar{P}}\right)>0$ & \\
\hline $\begin{array}{l}n \text { version, } \\
\text { humid }\end{array}$ & $\bar{P}>\overline{E_{0}}>\bar{E}$ & $\frac{\bar{P}}{\bar{E}}>\frac{\bar{P}}{\overline{E_{0}}}>1$ & $\ln \left(\frac{\bar{P}}{\bar{E}}\right)>\ln \left(\frac{\bar{P}}{\overline{E_{0}}}\right)>0$ & \\
\hline $\begin{array}{l}w \text { version, } \\
\text { arid }\end{array}$ & $\overline{E_{0}}>\bar{P}>\bar{E}$ & $\frac{\overline{E_{0}}+\bar{P}-\bar{E}}{\bar{P}}>\frac{\overline{E_{0}}}{\bar{P}}>1$ & $\ln \left(\frac{\overline{E_{0}}+\bar{P}-\bar{E}}{\bar{P}}\right)>\ln \left(\frac{\overline{E_{0}}}{\bar{P}}\right)$ & $>0$ \\
\hline $\begin{array}{l}w \text { version, } \\
\text { humid }\end{array}$ & $\bar{P}>\overline{E_{0}}>\bar{E}$ & $\frac{\bar{P}+\overline{E_{0}}-\bar{E}}{\overline{E_{0}}}>\frac{\bar{P}}{\overline{E_{0}}}>1$ & $\ln \left(\frac{\bar{P}+\overline{E_{0}}-\bar{E}}{\overline{E_{0}}}\right)>\ln \left(\frac{\bar{P}}{\overline{E_{0}}}\right)$ & $>0$ \\
\hline
\end{tabular}

\section{Properties of Analytical Expressions for $n$ and $w$}

Here we investigate the mathematical properties of Eq. (14) and (15) to determine that they are valid analytical expressions for $n$ and $w$. First, we examine the behavior of Eq. (15) as $\bar{E} \rightarrow 0$ and $\bar{E} \rightarrow \overline{E_{0}}$ or $\bar{P}$. Mathematically, the values of $n$ are constrained between 0 and $\infty$ (Yang et al., 2008), and the values of $w$ are constrained between 1 and $\infty$ (Zhang et al., 2004). Therefore, the upper and lower limits of the $n$ and $w$ versions of Eq. (15) should be equal to these respective constraints.

115 The lower limit for the $n$ version of Eq. (15),

$\lim _{\bar{E} \rightarrow 0} n=\frac{\ln (2)}{\infty}=0$,

and the upper limit,

$\lim _{E \rightarrow E_{0}} n=\lim _{E \rightarrow P} n=\frac{\ln (2)}{0}=\infty$, 
are equal to the lower and upper constraint for $n$, respectively. Similarly, the lower limit for the $w$ version of Eq. (15),

$120 \lim _{\bar{E} \rightarrow 0} w=\frac{\ln (2)}{\ln (2-0)}=1$,

and the upper limit,

$\lim _{\bar{E} \rightarrow \bar{E}_{0}} n=\lim _{\bar{E} \rightarrow \bar{P}} n=\frac{\ln (2)}{\ln (2-1)}=\frac{\ln (2)}{0}=\infty$,

are equal to the lower and upper constraint for $w$, respectively.

Next, we investigate the properties of Eq. (14). This equation contains a convergent infinite series whose value asymptotically approaches $e^{n}$ or $e^{w}$ for the $n$ and $w$ versions, respectively. The asymptotic behavior of the series' terms (e.g., monotonically decreasing or alternating sign and absolute value decreasing) depends on the specific values of $\bar{E}, \bar{P}$, and $\overline{E_{0}}$ (Fig. 1-4). To verify that both the $n$ and $w$ versions of Eq. (14) produce the correct values of $n$ and $w$ for a given set of $\bar{E}, \bar{P}$, and $\overline{E_{0}}$, we numerically invert Eq. (4) and (5) and compare the fitted $n$ and $w$ values, $\check{n}$ and $\breve{w}$, to successively better approximations of Eq. (14). The numerical inversion of Eq. (4) and (5) consists of numerically solving,

$130\left[\frac{\frac{\overline{E_{0}}}{\bar{P}}}{\left[1+\left(\overline{\frac{E_{0}}{\bar{P}}}\right)^{\breve{n}}\right]^{\frac{1}{n}}}-\frac{\bar{E}}{\bar{P}}\right]^{2}=0$.

and

$\left[1+\frac{\overline{E_{0}}}{\bar{P}}-\left(1+\left(\frac{\overline{E_{0}}}{\bar{P}}\right)^{\breve{W}}\right)^{\frac{1}{\breve{W}}}-\frac{\bar{E}}{\bar{P}}\right]^{2}=0$.

for $\breve{n}$ and $\breve{w}$, respectively. We compute approximations of Eq. (14) by truncating the infinite series to a finite number of terms, $N_{r}$. We successively improve these finite approximations by increasing $N_{r}$. As the number of terms in the finite series increase,

135 the approximations asymptotically converge to the $\breve{n}$ and $\breve{w}$ values obtained from the numerical inversion of Eq. (4) and (5) for both arid and humid climates (Fig. 1-4). This convergence is rapid (requiring fewer than ten terms) for typical values of $n$ and $w$ (i.e., $<4$ ) and provides strong numeric evidence that Eq. (14) yields valid analytical expressions for $n$ and $w$. 


$$
\frac{\bar{E}}{\bar{P}}=0.7
$$

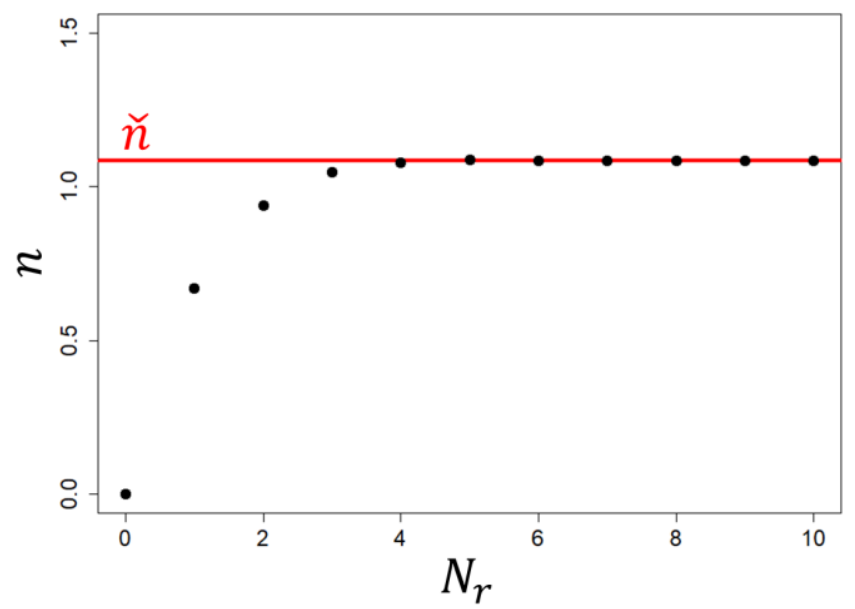

$\frac{\bar{E}}{\bar{P}}=0.1$

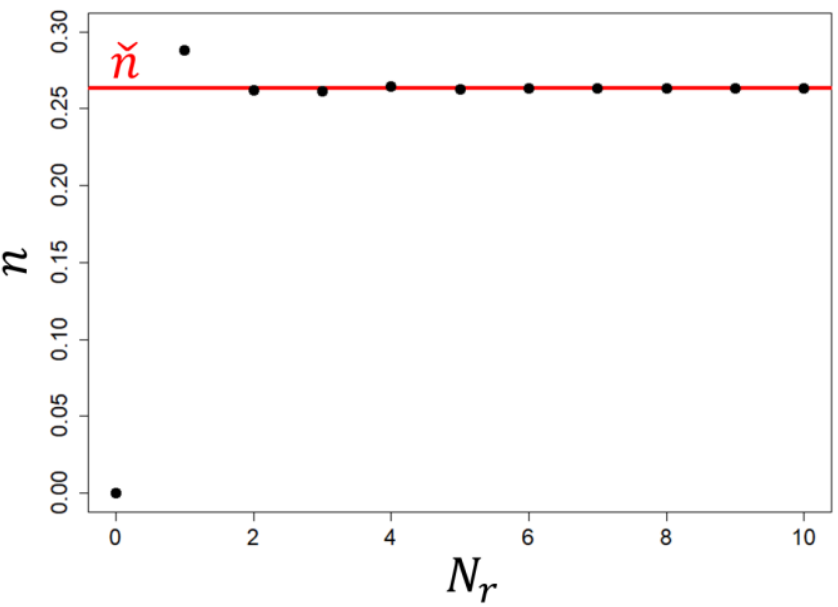

Figure 1: Comparison of $\breve{n}$ (red lines) from Eq. (20) to finite series approximations of $n$ (black dots) from Eq. (14) for increasing values of $N_{r}$ and an arid catchment $\left(\frac{\overline{E_{0}}}{\bar{P}}=2\right.$ ), with $\frac{\bar{E}}{\bar{P}}=0.7$ (left panel) and $\frac{\bar{E}}{\bar{P}}=0.1$ (right panel). In both cases, the finite series approximations of $n$ asymptotically converge to $\check{n}$ with increasing numbers of terms.

$$
\frac{\bar{E}}{\bar{P}}=0.7
$$

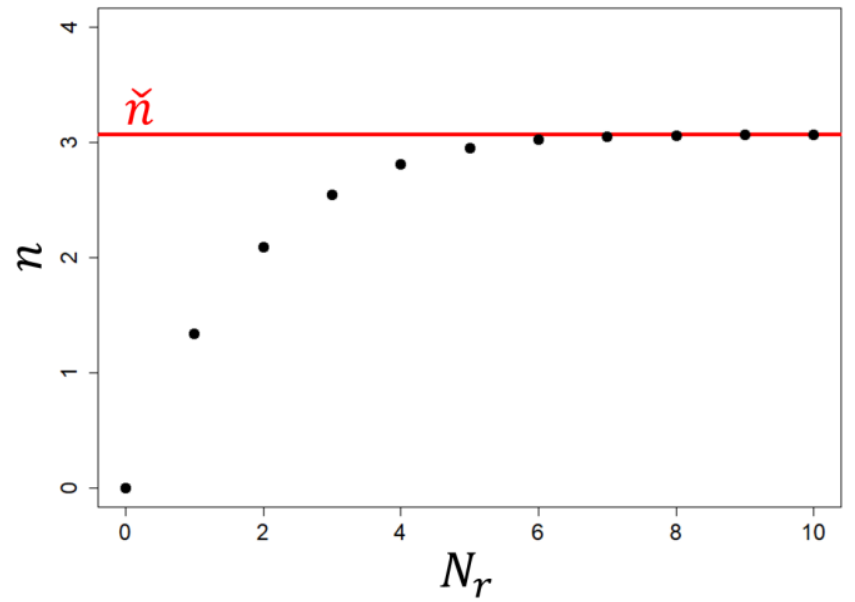

$\frac{\bar{E}}{\bar{P}}=0.02$

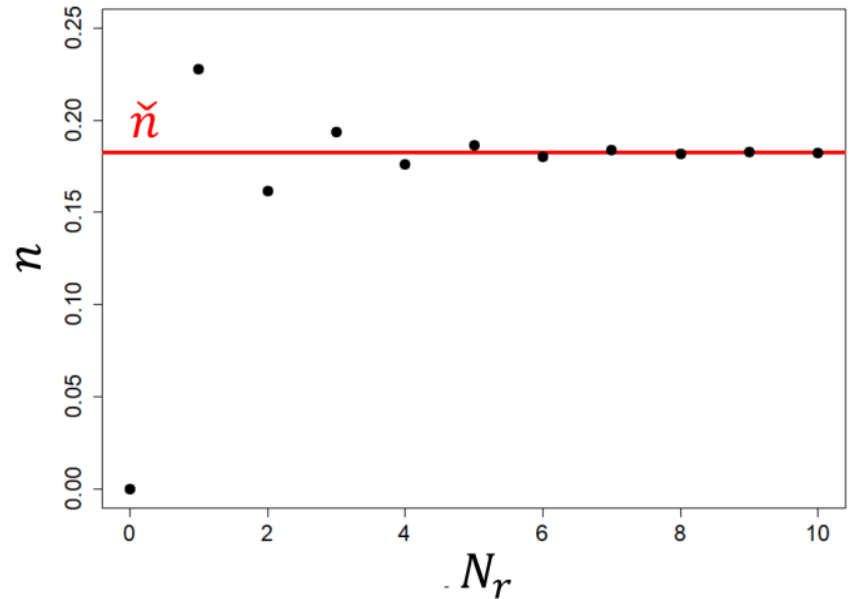

Figure 2: Comparison of $\breve{n}$ (red lines) from Eq. (20) to finite series approximations of $n$ (black dots) from Eq. (14) for increasing values of $N_{r}$ and a humid catchment $\left(\frac{\overline{E_{0}}}{\bar{P}}=0.8\right.$ ), with $\frac{\bar{E}}{\bar{P}}=0.7$ (left panel) and $\frac{\bar{E}}{\bar{P}}=0.02$ (right panel). In both cases, the finite series approximations of $n$ asymptotically converge to $\check{n}$ with increasing numbers of terms. 

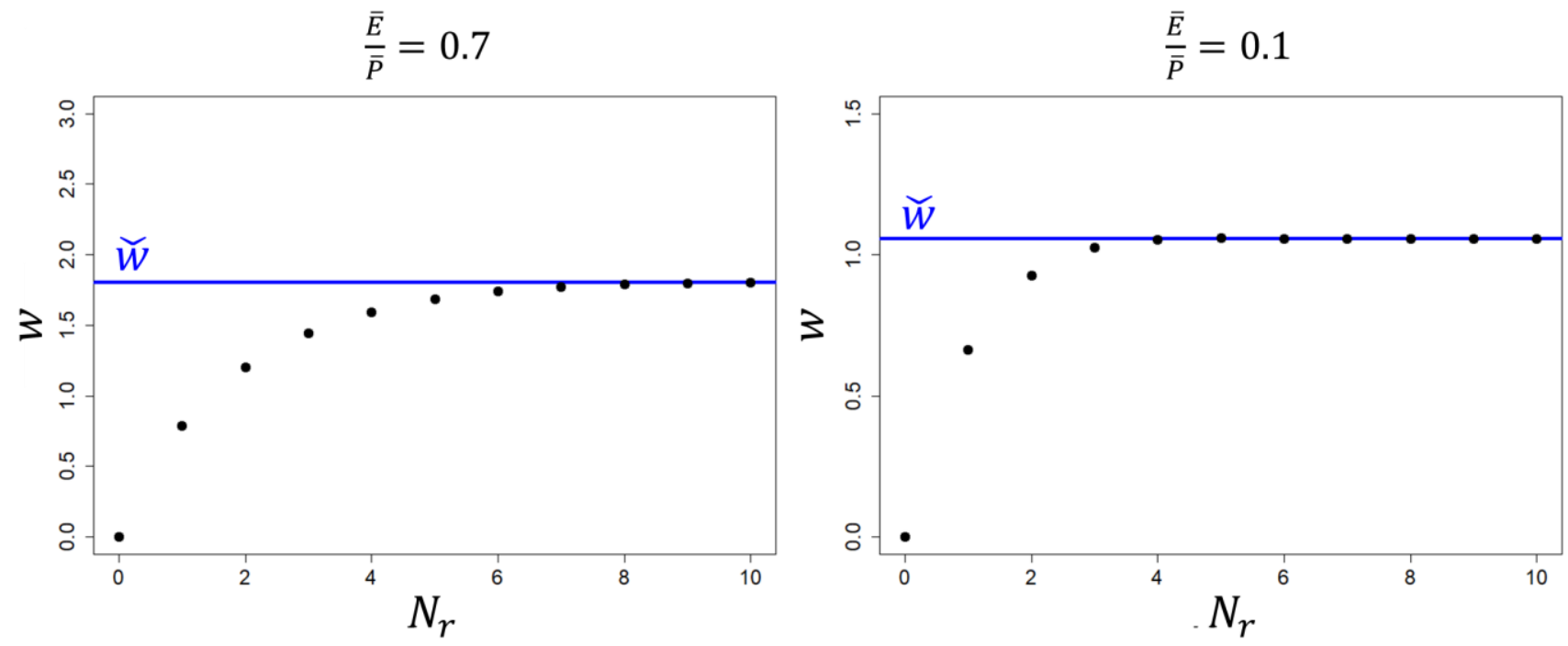

Figure 3: Comparison of $\breve{w}$ (blue lines) from Eq. (21) to finite series approximations of $w$ (black dots) from Eq. (14) for increasing values of $N_{r}$ and an arid catchment $\left(\frac{\overline{E_{0}}}{\bar{P}}=2\right.$ ), with $\frac{\bar{E}}{\bar{P}}=0.7$ (left panel) and $\frac{\bar{E}}{\bar{P}}=0.1$ (right panel). In both cases, the finite series approximations of $w$ asymptotically converge to $\breve{w}$ with increasing numbers of terms.

$$
\frac{\bar{E}}{\bar{P}}=0.7
$$

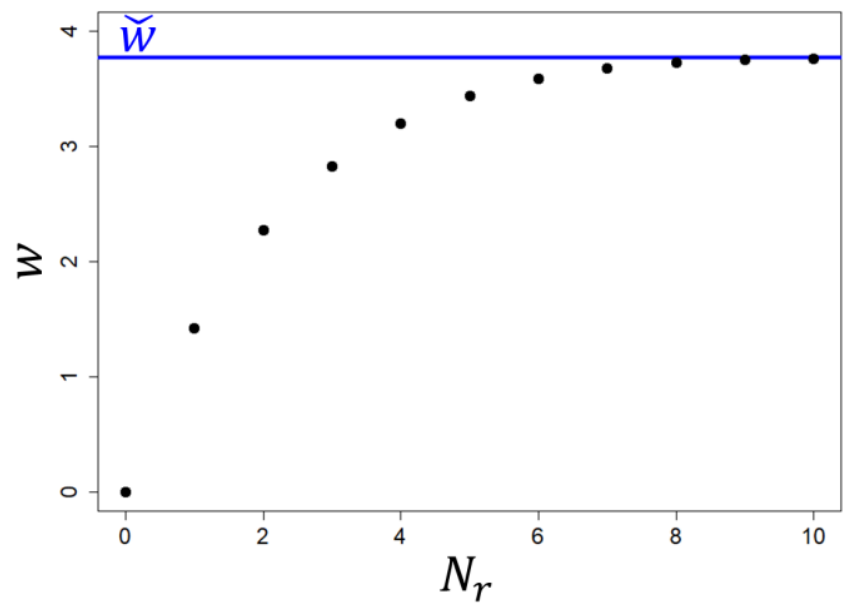

$\frac{\bar{E}}{\bar{P}}=0.02$

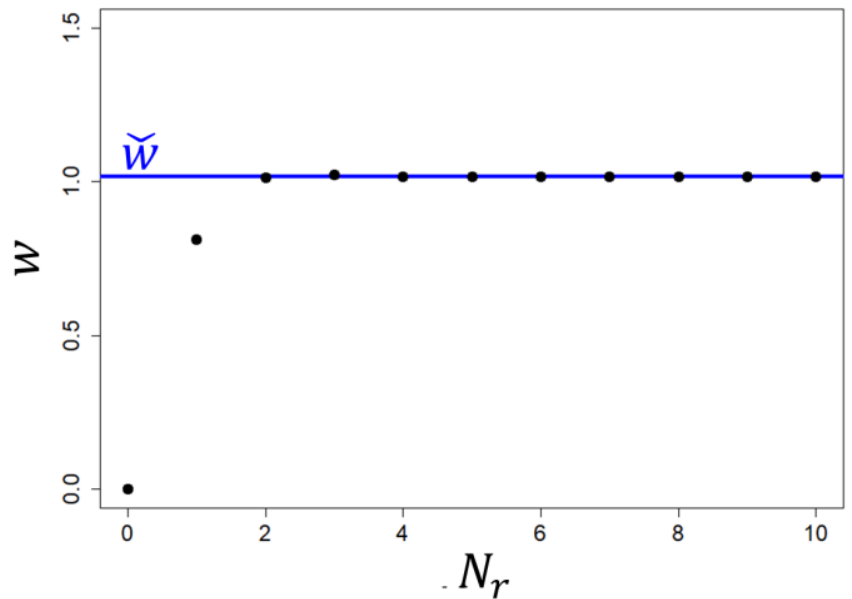

Figure 4: Comparison of $\breve{w}$ (blue lines) from Eq. (21) to finite series approximations of $w$ (black dots) from Eq. (14) for increasing values of $N_{r}$ and a humid catchment $\left(\frac{\overline{E_{0}}}{\bar{P}}=0.8\right.$ ), with $\frac{\bar{E}}{\bar{P}}=0.7$ (left panel) and $\frac{\bar{E}}{\bar{P}}=0.02$ (right panel). In both cases, the finite series approximations of $w$ asymptotically converge to $\breve{w}$ with increasing numbers of terms. 


\section{Discussion and Conclusion}

Inspection of Eq. (14) highlights the ambiguous nature of $n$ and $w$. The relationships between $n, w, \bar{E}, \overline{E_{0}}$, and $\bar{P}$ are fairly complicated, including logarithms of infinite series for the non-redundant cases. This highly nonlinear relationship challenges an intuitive understanding of how $n$ and $w$ should vary with changes to $\bar{E}, \overline{E_{0}}$, or $\bar{P}$ and is likely part of the reason why attempts to relate the catchment-specific parameter to climate and landscape features have yielded such divergent results (e.g., (Yang et al., 2007; Donohue et al., 2012; Yang et al., 2009; Shao et al., 2012; Li et al., 2013; Xu et al., 2013; Cong et al., 2015; Yang et al., 2016; Zhang et al., 2018; Abatzoglou and Ficklin, 2017; Xing et al., 2018; Zhao et al., 2020; Ning et al., 2020b; Ning et al., 2020a; Li et al., 2020b; Li et al., 2020a; Zhang et al., 2019; Ning et al., 2019; Bai et al., 2019; Ning et al., 2017)).

Notably, the explicit analytical expression for $n$ and $w$ from Eq. (14) illustrates that the value of the catchmentspecific parameter is only determined by $\bar{E}, \overline{E_{0}}$, and $\bar{P}$. Therefore, if $n$ or $w$ depend on biophysical features, it is directly due to the dependence of $\bar{E}, \overline{E_{0}}$, or $\bar{P}$ on those features. In short, this means that Eq. (14) is the general solution for how $n$ and $w$ depend on biophysical features. By substituting $\bar{E}, \overline{E_{0}}$, or $\bar{P}$ as functions of specific biophysical features into Eq. (14), one obtains the expression for $n$ and $w$ as a function of those features. Eq. (14) thus fulfills the literature-identified need of an analytical expression for $n$ and $w$ in terms of biophysical features.

\section{Author contributions}

NGFR conceived the study, performed the analytical inversion and analyses, and drafted the manuscript. All authors contributed in the interpretation of results and manuscript preparation.

\section{Competing interests}

175 The authors declare no conflicts of interest with respect to the results of this manuscript.

\section{Acknowledgments}

NGFR would like to acknowledge support from the University of Florida Graduate Fellowship.

\section{References}

Abatzoglou, J. T., and Ficklin, D. L.: Climatic and physiographic controls of spatial variability in surface water balance over the contiguous 180 United States using the Budyko relationship, Water Resources Research, 53, 7630-7643, 2017.

Bai, P., Liu, X., Zhang, D., and Liu, C.: Estimation of the Budyko model parameter for small basins in China, Hydrological Processes, 34, 125-138, 10.1002/hyp.13577, 2019. 
Berkaloff, E., and Tixeront, J.: Notice sur la carte du ruissellement annuel moyen en Tunisie, Études d'hydraulique et d'hydrologie, BIRH, 1958.

185 Budyko, M., and Zubenok, L.: The determination of evaporation from the land surface, Izv. Akad. Nauk SSSR Ser. Geogr, 6, 3-17, 1961. Choudhury, B.: Evaluation of an empirical equation for annual evaporation using field observations and results from a biophysical model, Journal of Hydrology, 216, 99-110, 1999.

Cong, Z., Zhang, X., Li, D., Yang, H., and Yang, D.: Understanding hydrological trends by combining the Budyko hypothesis and a stochastic soil moisture model, Hydrological Sciences Journal, 60, 145-155, 10.1080/02626667.2013.866710, 2015.

190 Donohue, R. J., Roderick, M. L., and McVicar, T. R.: Roots, storms and soil pores: Incorporating key ecohydrological processes into Budyko's hydrological model, Journal of Hydrology, 436-437, 35-50, 10.1016/j.jhydrol.2012.02.033, 2012.

$\mathrm{Fu}, \mathrm{B} .:$ On the calculation of the evaporation from land surface, Sci. Atmos. Sin, 5, 23-31, 1981.

Gentine, P., D'Odorico, P., Lintner, B. R., Sivandran, G., and Salvucci, G.: Interdependence of climate, soil, and vegetation as constrained by the Budyko curve, Geophysical Research Letters, 39, n/a-n/a, 10.1029/2012g1053492, 2012.

195 Greve, P., Gudmundsson, L., Orlowsky, B., and Seneviratne, S. I.: Introducing a probabilistic Budyko framework, Geophysical Research Letters, 42, 2261-2269, 10.1002/2015g1063449, 2015.

Hochstadt, H.: The functions of mathematical physics, Courier Corporation, 2012.

Hulme, M., Marsh, R., and Jones, P. D.: Global changes in a humidity index between 1931-60 and 1961-90, Climate Research, 2, 1-22, 10.3354/cr002001, 1992.

200 Li, D., Pan, M., Cong, Z., Zhang, L., and Wood, E.: Vegetation control on water and energy balance within the Budyko framework, Water Resources Research, 49, 969-976, 10.1002/wrcr.20107, 2013.

Li, S., Zhang, L., Du, Y., Zhuang, Y., and Yan, C.: Anthropogenic Impacts on Streamflow-Compensated Climate Change Effect in the Hanjiang River Basin, China, Journal of Hydrologic Engineering, 25, 10.1061/(asce)he.1943-5584.0001876, 2020a.

Li, T., Xia, J., She, D., Cheng, L., Zou, L., and Liu, B.: Quantifying the Impacts of Climate Change and Vegetation Variation on Actual

205 Evapotranspiration Based on the Budyko Hypothesis in North and South Panjiang Basin, China, Water, 12, 10.3390/w12020508, 2020b.

Mezentsev, V.: More on the calculation of average total evaporation, Meteorol. Gidrol, 5, 24-26, 1955.

Milly, P. C. D., Kam, J., and Dunne, K. A.: On the Sensitivity of Annual Streamflow to Air Temperature, Water Resources Research, 54, 2624-2641, doi:10.1002/2017WR021970, 2018.

Ning, T., Li, Z., and Liu, W.: Vegetation dynamics and climate seasonality jointly control the interannual catchment water balance in the

210 Loess Plateau under the Budyko framework, Hydrology and Earth System Sciences, 21, 1515-1526, 10.5194/hess-21-1515-2017, 2017.

Ning, T., Zhou, S., Chang, F., Shen, H., Li, Z., and Liu, W.: Interaction of vegetation, climate and topography on evapotranspiration modelling at different time scales within the Budyko framework, Agricultural and Forest Meteorology, 275, 59-68, 10.1016/j.agrformet.2019.05.001, 2019.

Ning, T., Li, Z., Feng, Q., Chen, W., and Li, Z.: Effects of forest cover change on catchment evapotranspiration variation in China,

215 Hydrological Processes, 34, 2219-2228, 10.1002/hyp.13719, 2020a.

Ning, T., Liu, W., Li, Z., and Feng, Q.: Modelling and attributing evapotranspiration changes on China's Loess Plateau with Budyko framework considering vegetation dynamics and climate seasonality, Stochastic Environmental Research and Risk Assessment, 10.1007/s00477-020-01813-0, 2020b.

Ol'Dekop, E.: On evaporation from the surface of river basins, Transactions on meteorological observations, 4, $200,1911$.

220 Reaver, N. G. F., Kaplan, D. A., Klammler, H., and Jawitz, J. W.: Reinterpreting the Budyko Framework, Hydrology \& Earth System Sciences, 2020, (submitted 10 November 2020).

Roderick, M. L., and Farquhar, G. D.: A simple framework for relating variations in runoff to variations in climatic conditions and catchment properties, Water Resources Research, 47, n/a-n/a, 10.1029/2010wr009826, 2011.

Schreiber, P.: Über die Beziehungen zwischen dem Niederschlag und der Wasserführung der Flüsse in Mitteleuropa, Z. Meteorol, 21, 441-

\section{$225452,1904$.}

Shao, Q., Traylen, A., and Zhang, L.: Nonparametric method for estimating the effects of climatic and catchment characteristics on mean annual evapotranspiration, Water Resources Research, 48, 10.1029/2010wr009610, 2012.

Shen, Q., Cong, Z., and Lei, H.: Evaluating the impact of climate and underlying surface change on runoff within the Budyko framework: A study across 224 catchments in China, Journal of Hydrology, 554, 251-262, 10.1016/j.jhydrol.2017.09.023, 2017.

230 Tixeront, J.: Prévision des apports des cours d'eau, Symposium sur les Eau de surface, Assemblée générale de l'IUGG, Berkeley, 1964, Turc, L.: Le bilan d'eau des sols: relations entre les précipitations, l'évaporation et l'écoulement, 1953.

Wang, C., Wang, S., Fu, B., and Zhang, L.: Advances in hydrological modelling with the Budyko framework: A review, Progress in Physical Geography, 40, 409-430, 10.1177/0309133315620997, 2016a.

Wang, D., and Hejazi, M.: Quantifying the relative contribution of the climate and direct human impacts on mean annual streamflow in the

235 contiguous United States, Water Resources Research, 47, n/a-n/a, 10.1029/2010wr010283, 2011.

Wang, W., Zou, S., Shao, Q., Xing, W., Chen, X., Jiao, X., Luo, Y., Yong, B., and Yu, Z.: The analytical derivation of multiple elasticities of runoff to climate change and catchment characteristics alteration, Journal of Hydrology, 541, 1042-1056, 10.1016/j.jhydrol.2016.08.014, 2016 b. 
Xing, W., Wang, W., Shao, Q., and Yong, B.: Identification of dominant interactions between climatic seasonality, catchment characteristics

240 and agricultural activities on Budyko-type equation parameter estimation, Journal of Hydrology, 556, 585-599, 10.1016/j.jhydrol.2017.11.048, 2018.

Xu, X., Liu, W., Scanlon, B. R., Zhang, L., and Pan, M.: Local and global factors controlling water-energy balances within the Budyko framework, Geophysical Research Letters, 40, 6123-6129, 10.1002/2013g1058324, 2013.

Yang, D., Sun, F., Liu, Z., Cong, Z., Ni, G., and Lei, Z.: Analyzing spatial and temporal variability of annual water-energy balance in 245 nonhumid regions of China using the Budyko hypothesis, Water Resources Research, 43, n/a-n/a, 10.1029/2006wr005224, 2007.

Yang, D., Shao, W., Yeh, P. J. F., Yang, H., Kanae, S., and Oki, T.: Impact of vegetation coverage on regional water balance in the nonhumid regions of China, Water Resources Research, 45, 10.1029/2008wr006948, 2009.

Yang, H., Yang, D., Lei, Z., and Sun, F.: New analytical derivation of the mean annual water-energy balance equation, Water Resources Research, 44, n/a-n/a, 10.1029/2007wr006135, 2008.

250 Yang, H., and Yang, D.: Derivation of climate elasticity of runoff to assess the effects of climate change on annual runoff, Water Resources Research, 47, 10.1029/2010wr009287, 2011.

Yang, Y., Donohue, R. J., and McVicar, T. R.: Global estimation of effective plant rooting depth: Implications for hydrological modeling, Water Resources Research, 52, 8260-8276, 10.1002/2016wr019392, 2016.

Yao, A. Y. M.: Agricultural potential estimated from the ratio of actual to potential evapotranspiration, Agricultural Meteorology, 13, 405-

255 417, https://doi.org/10.1016/0002-1571(74)90081-8, 1974.

Zhang, L., Hickel, K., Dawes, W. R., Chiew, F. H. S., Western, A. W., and Briggs, P. R.: A rational function approach for estimating mean annual evapotranspiration, Water Resources Research, 40, n/a-n/a, 10.1029/2003wr002710, 2004.

Zhang, S., Yang, H., Yang, D., and Jayawardena, A.: Quantifying the effect of vegetation change on the regional water balance within the Budyko framework, Geophysical Research Letters, 43, 1140-1148, 2016.

260 Zhang, S., Yang, Y., McVicar, T. R., and Yang, D.: An Analytical Solution for the Impact of Vegetation Changes on Hydrological Partitioning Within the Budyko Framework, Water Resources Research, n/a-n/a, 10.1002/2017WR022028, 2018.

Zhang, X., Dong, Q., Costa, V., and Wang, X.: A hierarchical Bayesian model for decomposing the impacts of human activities and climate change on water resources in China, Sci Total Environ, 665, 836-847, 10.1016/j.scitotenv.2019.02.189, 2019.

Zhao, J., Huang, S., Huang, Q., Leng, G., Wang, H., and Li, P.: Watershed water-energy balance dynamics and their association with diverse

265 influencing factors at multiple time scales, Sci Total Environ, 711, 135189, 10.1016/j.scitotenv.2019.135189, 2020.

Zhou, S., Yu, B., Zhang, L., Huang, Y., Pan, M., and Wang, G.: A new method to partition climate and catchment effect on the mean annual runoff based on the Budyko complementary relationship, Water Resources Research, 10.1002/2016wr019046, 2016.

\section{Appendix A: Details of Analytical Inversion}

270 Here we develop analytical inversions of the two parametric Budyko equations. The steps for these derivations are:

1) Produce a general form for both parametric forms of the Budyko equation.

2) Determine the Mellin transform for the general form.

3) Manipulate the Mellin transformed general form into functions with known inversions.

4) Take the inverse Mellin transform of the general form to find a general solution.

275 5) Substitute the specific functional forms of each parametric Budyko equation into the general solution to produce explicit expressions for $n$ and $w$.

The outline for portions of these derivations was developed from Hochstadt (2012), p. 81-84.

\section{A.1 Producing a general form for both parametric forms of the Budyko equation}

Equation (4) of the main text can be rearranged in the following manner,

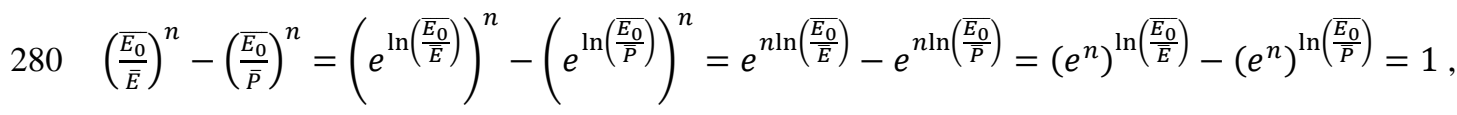


to produce Eq. (8). Following the same procedure, Eq. (5), (6), and (7) can be rearranged to Eq. (9), (10), and (11). The general form of Eq. (8), (9), (10), and (11) is Eq. (12). We use the Eq. (12) and the condition, $C>D>0$ (Table 1), to derive a general solution which will allow for specific solutions of $n$ and $w$.

\section{A.2 The Mellin transform for $y(z)$}

285 The Mellin transform for $y(z)$ of Eq. (12) is,

$Y(s)=\int_{0}^{\infty} z^{s-1} y(z) d z$

where $s$ is a complex number. Whether the improper integral in Eq. (A2) converges or diverges depends on the behavior of $y(z)$ and the value of $s$. Letting $z=0$ in Eq. (12) gives,

$y(0)=1$

290 Taking the first derivative of Eq. (12) gives,

$\frac{d y}{d z}=\frac{-y^{D}}{C y^{C-1}+z D y^{D-1}}$,

Since $C>D>0$, Eq. (A4) is always negative, meaning that $y(z)$ is a monotonically decreasing function for $0 \leq z<\infty$. Additionally, $y(z)=0$ is not a valid solution to Eq. (12), therefore, $1 \geq y(z)>0$ for $0 \leq z<\infty$. As $z \rightarrow \infty, y(z)$ will become very small. This allows us to rearrange Eq. (12) to get an approximate functional form for $y(z)$ for large values of $z$,

$295 y^{D}=\frac{1-y^{C}}{z} \approx \frac{1}{z}$,

therefore,

$y(z) \approx z^{-\frac{1}{D}}$.

Thus, from Eq. (A3), (A4), and (A6), the integrand of Eq. (A2) is $\approx z^{s-1}$ for small values of $z$ and transitions to $\approx z^{s-\frac{1}{D}-1}$ for as $z$ increases. Splitting Eq. (A2) into two component integrals and substituting in Eq. (A3) for small values of $z$ and Eq. (A6)

300 for large values of $z$ gives,

$\int_{0}^{\infty} z^{s-1} y(z) d z=\int_{0}^{1} z^{s-1} y(z) d z+\int_{1}^{\infty} z^{s-1} y(z) d z \approx \int_{0}^{1} z^{s-1} d z+\int_{1}^{\infty} z^{s-\frac{1}{D}-1} d z$.

To find the values of $s$ for which Eq. (A2) converges, we determine the values of $s$ for which the two approximate integrals in Eq. (A7) converge. Integrating Eq. (A7), and expressing $s$ as the sum of its real and imaginary parts, $s=a+b i$ where $i=$ $\sqrt{-1}$, yields,

$305 \int_{0}^{1} z^{s-1} d z+\int_{1}^{\infty} z^{s-\frac{1}{D}-1} d z=\left[\frac{z^{s}}{s}\right]_{0}^{1}+\left[\frac{z^{s-\frac{1}{D}}}{s-\frac{1}{D}}\right]_{1}^{\infty}=\left[\frac{z^{a+b i}}{a+b i}\right]_{0}^{1}+\left[\frac{z^{a+b i-\frac{1}{D}}}{a+b i-\frac{1}{D}}\right]_{1}^{\infty}=\left[\frac{z^{b i} z^{a}}{a+b i}\right]_{0}^{1}+\left[\frac{z^{b i} z^{a-\frac{1}{D}}}{a+b i-\frac{1}{D}}\right]_{1}^{\infty}$.

Rearranging Eq. (A8) and using Euler's formula, $e^{i x}=\cos (x)+i \sin (x)$, yields, 
$\left[\frac{e^{i b \ln (z)} z^{a}}{a+b i}\right]_{0}^{1}+\left[\frac{e^{i b \ln (z)} z^{a-\frac{1}{D}}}{a+b i-\frac{1}{D}}\right]_{1}^{\infty}=\left[\frac{[\cos (b \ln (z))+i \sin (b \ln (z))] z^{a}}{a+b i}\right]_{0}^{1}+\left[\frac{[\cos (b \ln (z))+i \sin (b \ln (z))] z^{a-\frac{1}{D}}}{a+b i-\frac{1}{D}}\right]_{1}^{\infty}$.

The value of the expression, $\cos (b \ln (z))+i \sin (b \ln (z))$, in Eq. (A9) is bounded to a circular region of radius 1 around the origin in the complex plane, making it finite for all value of $z$. Evaluating Eq. (A9) and $\operatorname{setting} F(z)=\cos (b \ln (z))+$ $310 i \sin (b \ln (z))$ to represent its finite value, gives,

$\left[\frac{F(z) z^{a}}{a+b i}\right]_{0}^{1}+\left[\frac{F(z) z^{a-\frac{1}{D}}}{a+b i-\frac{1}{D}}\right]_{1}^{\infty}=\frac{F(1)}{a+b i}-\frac{F(0)}{a+b i} \lim _{z \rightarrow 0} z^{a}+\frac{F(\infty)}{a+b i-\frac{1}{D}} \lim _{z \rightarrow \infty} z^{a-\frac{1}{D}}-\frac{F(1)}{a+b i-\frac{1}{D}}$.

If both limits of Eq. (A10), $\lim _{z \rightarrow 0} z^{a}$ and $\lim _{z \rightarrow \infty} z^{a-\frac{1}{D}}$, exist, then Eq. (A2) will be convergent. The first of these limits, $\lim _{z \rightarrow 0} z^{a}$, will give a finite value if,

$a>0$

315 The second limit, $\lim _{z \rightarrow \infty} z^{a-\frac{1}{D}}$, will give a finite value if,

$a-\frac{1}{D}<0$,

which gives,

$a<\frac{1}{D}$

Thus, Eq. (A2) converges and the Mellin transform exists if,

$0<\operatorname{Re}(s)<\frac{1}{D}$

\section{A.3 Mellin transformed $y(z)$ in terms of known functions}

Next, we evaluate Eq. (A2) explicitly. To do this, we switch the integration from z to y, using Eq. (12). This involves expressing $\mathrm{z}$ in terms of $y$,

$z=y^{-D}-y^{C-D}$

325 expressing $d z$ in terms of $\mathrm{y}$ and $d y$,

$d z=-\left[D y^{-D-1}+(C-D) y^{C-D-1}\right] d y$,

and expressing the limits of integration in terms of $y$,

when $z=0, \quad y=1$

when $z \rightarrow \infty, \quad y \rightarrow 0$.

We can now rewrite Eq. (A2) in terms of a $y$ integration, 
$330 Y(s)=-\int_{1}^{0} y\left(y^{-D}-y^{C-D}\right)^{s-1}\left[D y^{-D-1}+(C-D) y^{C-D-1}\right] d y$,

which can be rearranged to,

$Y(s)=D \int_{0}^{1} y^{-D s}\left(1-y^{C}\right)^{s-1} d y+(C-D) \int_{0}^{1} y^{-D s+C}\left(1-y^{C}\right)^{s-1} d y$.

Making the substitution $y^{C}=u$, we have,

$d y=\frac{1}{C} y^{-C+1} d u=\frac{1}{C} u^{\frac{1}{c}-1} d u$,

335 while the limits of integration remain the same,

when $y=0, \quad u=0$

when $y=1, u=1$,

therefore, Eq. (A19) becomes,

$Y(s)=\frac{D}{C} \int_{0}^{1} u^{\frac{1-D s}{C}-1}(1-u)^{s-1} d u+\frac{C-D}{C} \int_{0}^{1} u^{\frac{1-D s}{C}+1-1}(1-u)^{s-1} d u$.

The integrals in Eq. (A22) are of the same form as the integral definition of the Beta function, allowing $Y(s)$ to be expressed 340 as a sum of Beta functions,

$Y(s)=\left[\frac{D}{C}\right] B\left(\frac{1-D s}{C}, s\right)+\left[\frac{C-D}{C}\right] B\left(\frac{1-D s}{C}+1, s\right)$,

where $B($,$) is the Beta function. The Beta function in turn can also be defined in terms of the Gamma function (i.e. B(q, L)=$ $\frac{\Gamma(q) \Gamma(L)}{\Gamma(q+L)}$ ), allowing Eq. (A23) to be rewritten as,

$Y(s)=\left[\frac{D}{C}\right] \frac{\Gamma\left(\frac{1-D s}{C}\right) \Gamma(s)}{\Gamma\left(\frac{1-D s}{C}+s\right)}+\left[\frac{C-D}{C}\right] \frac{\Gamma\left(\frac{1-D s}{C}+1\right) \Gamma(s)}{\Gamma\left(\frac{1-D s}{C}+1+s\right)}$,

345 where $\Gamma()$ is the Gamma function. The Gamma function has the property $\Gamma(q+1)=q \Gamma(q)$, which allows Eq. (A24) to be simplified to,

$Y(s)=\left[\frac{\Gamma(s)}{C}\right]\left[\frac{D\left(\frac{1-D s}{C}+s\right) \Gamma\left(\frac{1-D s}{C}\right)}{\Gamma\left(\frac{1-D s}{C}+1+s\right)}+\frac{(C-D)\left(\frac{1-D s}{C}\right) \Gamma\left(\frac{1-D s}{C}\right)}{\Gamma\left(\frac{1-D s}{C}+1+s\right)}\right]$,

and,

$Y(s)=\left[\frac{\Gamma(s)}{C}\right]\left[\frac{s D \Gamma\left(\frac{1-D s}{C}\right)+C\left(\frac{1-D s}{C}\right) \Gamma\left(\frac{1-D s}{C}\right)}{\Gamma\left(\frac{1-D s}{C}+1+s\right)}\right]=\left[\frac{\Gamma(s) \Gamma\left(\frac{1-D s}{C}\right)}{C}\right]\left[\frac{1-D s+D s}{\Gamma\left(\frac{1-D s}{C}+1+s\right)}\right]=\frac{\Gamma(s) \Gamma\left(\frac{1-D s}{C}\right)}{C \Gamma\left(\frac{1-D s}{C}+1+s\right)}$. 


\section{A.4 The inverse Mellin transform and solution for $y(z)$}

We now take the inverse Mellin transform of Eq. (A26) and solve for $y(z)$ explicitly. The inverse Mellin transform is defined as,

$y(z)=\frac{1}{2 \pi i} \int_{k-\infty i}^{k+\infty i} Y(s) z^{-s} d s$,

where the integral from $k-\infty i$ to $k+\infty i$ is interpreted as a line integral along a vertical line in the complex plane. For our specific function, the inverse Mellin transform is,

$y(z)=\frac{1}{2 \pi i} \int_{k-\infty i}^{k+\infty i} \frac{z^{-s} \Gamma(s) \Gamma\left(\frac{1-D s}{C}\right)}{C \Gamma\left(\frac{1-D s}{C}+1+s\right)} d s \quad$ where $0<k<\frac{1}{D}$.

The constraint $0<k<\frac{1}{D}$ is due to Eq. (A14), the constraint on the real part of $s$ so that Eq. (A2) would converge. This means that the vertical line in the complex plane over which the line integral is taken must fall between 0 and $\frac{1}{D}$ on the real axis (Fig. A1). We evaluate the integral in Eq. (A28) to find an explicit form of $y(z)$ using the following methodology:

1) Define an appropriate contour in the complex plane to perform a contour integration of $Y(s) Z^{-s}$.

2) Use residue integration to evaluate the value of this contour integral.

3) Show that the only part of this contour that does not vanish is the line integral defined in Eq. (A28), meaning the inverse Mellin transform, and therefore $y(z)$, is equal to the value of the contour integral evaluated in step 2.

First, we choose a semicircle contour in the complex plane, with the straight portion as a vertical line crossing the real axis at $s=k$, and the circular portion connecting the ends of this line across the left side of the complex plane (Fig. A1). This contour is consistent with the constraint on the real part of $s$ given in Eq. (A14). We can now define the integral of $Y(s) Z^{-s}$ over this contour,

$\Lambda(z)=\oint Y(s) z^{-s} d s$

$\Lambda(z)$ can be expressed as the sum of two line integrals, one over the vertical line portion of the contour, and one over the circular arc portion of the contour,

$\Lambda(z)=\oint Y(s) z^{-s} d s=\int_{k-R i}^{k+R i} Y(s) z^{-s} d s+\int_{a r c} Y(s) z^{-s} d s$,

where $R$ is the radius of the semicircle contour. Allowing $R \rightarrow \infty$ leads to,

$\Lambda(z)=\oint Y(s) z^{-s} d s=\int_{k-\infty i}^{k+\infty i} Y(s) z^{-s} d s+\int_{a r c} Y(s) z^{-s} d s$.

We can now evaluate $\Lambda(z)$ over the infinitely large contour using residue integration. Residue integration relates the value of a contour integral to the sum of the residues of the function being integrated. Residues occur when the function of interest has singularities within the contour. Inspection of $Y(s) Z^{-s}$ (i.e. the integrand in Eq. (A26)) inside the semicircle contour shows 
that the only component with singularities is $\Gamma(s) . \Gamma(s)$ is undefined and has simple poles at $s=-r$ where $r=0,1,2,3, \ldots \infty$. The residues of the gamma function for each value of $r$ are,

$\operatorname{Res}(\Gamma(s),-r)=\frac{(-1)^{r}}{r !}$

380 Using the residue theorem we can evaluate $\Lambda(z)_{2}$

$\Lambda(z)=\oint Y(s) z^{-s} d s=2 \pi i \sum \operatorname{Res}\left(Y(s) z^{-s},-r\right)=2 \pi i \sum_{r=0}^{\infty} \frac{z^{r}(-1)^{r} \Gamma\left(\frac{1+D r}{C}\right)}{r ! C \Gamma\left(\frac{1+D r}{C}+1-r\right)}$

which contains the sum over the infinite number of residues of $Y(s) z^{-s}$ within the semicircular contour. Substituting this solution into Eq. (A29) gives,

$\Lambda(z)=\int_{k-\infty i}^{k+\infty i} Y(s) z^{-s} d s+\int_{a r c} Y(s) z^{-s} d s=2 \pi i \sum_{r=0}^{\infty} \frac{z^{r}(-1)^{r} \Gamma\left(\frac{1+D r}{C}\right)}{r ! C \Gamma\left(\frac{1+D r}{C}+1-r\right)}$

385 Equation (A34) is the line integral of $Y(s) z^{-s}$ evaluated for the entire contour; now we investigate the contribution of the line integral over just the circular arc portion of the contour. Using the estimation lemma, we can write the following inequality for the line integral over the arc,

$\left|\int_{\operatorname{arc}} Y(s) z^{-s} d s\right| \leq M L$

where $L$ is the length of the arc and $M$ is the maximum value of $\left|Y(s) z^{-s}\right|$ along the length of the arc. Writing $s$ in terms of its real and imaginary parts, $s=a+b i$, the length of the arc is defined as the product of the central angle and radius of the circle,

$L=\pi R=\pi \sqrt{a^{2}+b^{2}}$.

$\left|Y(s) z^{-s}\right|$ can be written as,

$\left|Y(s) z^{-s}\right|=\frac{\left|z^{-s}\right||\Gamma(s)|\left|\Gamma\left(\frac{1-D s}{C}\right)\right|}{\left|C \Gamma\left(\frac{1-D s}{C}+1+s\right)\right|}$

We are interested in the value of $\left|Y(s) z^{-s}\right|$ as $R \rightarrow \infty$, therefore, we can approximate the absolute values of the gamma functions using a form of Stirling's formula, valid for values of $s$ as $|b| \rightarrow \infty$,

$|\Gamma(s)|=|\Gamma(a+b i)| \sim \sqrt{2 \pi} e^{\frac{-\pi|b|}{2}}|b|^{a-\frac{1}{2}}$.

Applying Stirling's approximation to Eq. (A37) yields,

$\left|Y(s) z^{-S}\right| \approx \frac{\left|z^{-a-b i}\right| \sqrt{2 \pi} e^{\frac{-\pi|b|}{2}|b|^{a-\frac{1}{2}} \sqrt{2 \pi} e^{\frac{-\pi\left|-\frac{D}{C} b\right|}{2}}\left|-\frac{D}{C} b\right|^{\frac{1-D}{C} a-\frac{1}{2}}}}{C \sqrt{2 \pi} e^{\frac{-\pi\left|\left(1-\frac{D}{C}\right) b\right|}{2}}\left|\left(1-\frac{D}{C}\right) b\right|^{\frac{C+1-D}{C} a+1-\frac{1}{2}}}$, 
which becomes,

400

$\left|Y(s) Z^{-S}\right| \approx \frac{\sqrt{2 \pi} z^{-a} e^{\left[\frac{-\pi}{2} b+\frac{-\pi D}{2} C+\frac{\pi}{2}\left(1-\frac{D}{C}\right) b\right]_{b}\left[a-\frac{C+1-D}{C} a+\frac{1-D}{C} a-\frac{3}{2}\right]\left(\frac{D}{C}\right)^{\left[\frac{1-D}{C} a-\frac{1}{2}\right]}}}{C\left(1-\frac{D}{C}\right)^{\left[\frac{C+1-D}{C} a+\frac{1}{2}\right]}}=\frac{\sqrt{\frac{2 \pi}{C D-D^{2}} z^{-a}} e^{\frac{-\pi D b}{C} b^{-\frac{3}{2}}\left(\frac{D}{C}\right)^{\left[\frac{1-D}{C} a\right]}}}{\left(1-\frac{D}{C}\right)^{\left[\frac{C+1-D}{C} a\right]}}$,

resulting in,

$\left|Y(s) z^{-s}\right| \approx\left[\sqrt{\frac{2 \pi}{C D-D^{2}}}\right]\left[\frac{\left(\frac{D}{C}\right)^{\left(\frac{1-D}{C}\right)}}{z\left(1-\frac{D}{C}\right)^{\left(\frac{C+1-D}{C}\right)}}\right]^{a}\left[e^{\frac{-\pi D b}{C}}\right]\left[b^{-\frac{3}{2}}\right]$.

Inspecting Eq. (A41), we see that $\left|Y(s) z^{-s}\right|$ decreases as $|b|$ increases and $\left|Y(s) z^{-s}\right|$ increases as $a$ increases. Therefore, $M$ occurs at the starting and ending point of the arc, where $a=k$. When $a=k$, letting $R \rightarrow \infty$ is equivalent to letting $|b| \rightarrow \infty$,

405 allowing us to evaluate $M L$,

$M L=\lim _{|b| \rightarrow \infty} \pi \sqrt{k^{2}+b^{2}}\left[\sqrt{\frac{2 \pi}{C D-D^{2}}}\right]\left[\frac{\left(\frac{D}{C}\right)^{\left(\frac{1-D}{C}\right)}}{z\left(1-\frac{D}{C}\right)}\right]^{k} \frac{\left(\frac{C+1-D}{C}\right)}{k}\left[e^{\frac{-\pi D b}{C}}\right]\left[b^{-\frac{3}{2}}\right]=0$.

Equation (A42) implies that the contribution of the circular arc portion of the contour vanishes,

$0 \leq\left|\int_{\operatorname{arc}} Y(s) z^{-s} d s\right| \leq M L=0$,

which means,

$410 \int_{\operatorname{arc}} Y(s) z^{-s} d s=\left|\int_{\operatorname{arc}} Y(s) z^{-s} d s\right|=0$,

and therefore,

$y(z)=\frac{1}{2 \pi i} \Lambda(z)=\frac{1}{2 \pi i} \int_{k-\infty i}^{k+\infty i} Y(s) z^{-s} d s=\sum_{r=0}^{\infty} \frac{z^{r}(-1)^{r} \Gamma\left(\frac{1+D r}{C}\right)}{r ! C \Gamma\left(\frac{1+D r}{C}+1-r\right)}$,

which is the solution for the general form, Eq. (12). 


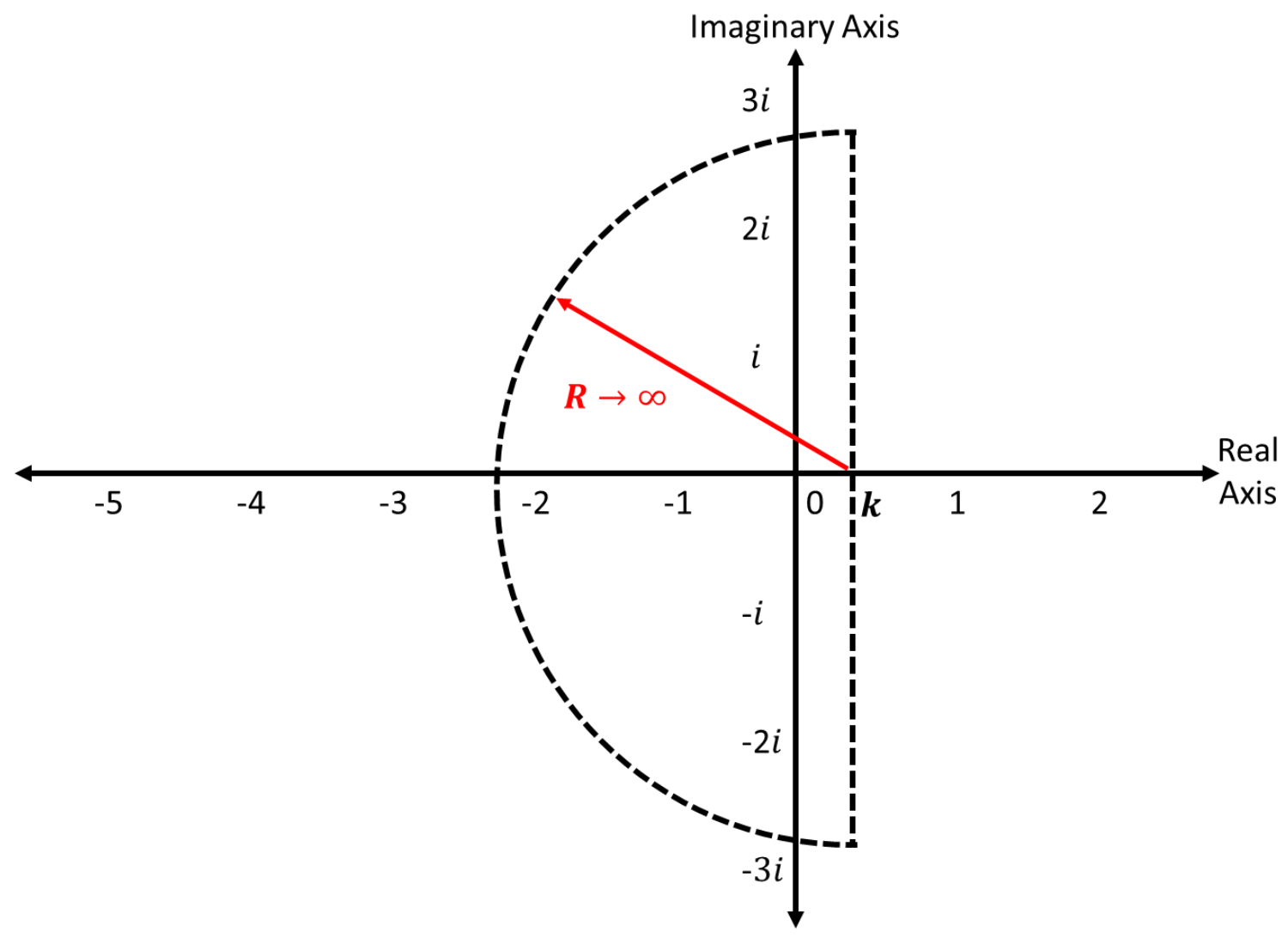

415 Figure A1: Illustration of the semicircular contour in the complex plane, used to evaluate Eq. (A28). The contour is composed of a vertical line crossing the real axis at $s=k$ and an arc connecting the two ends of the vertical line. The radius of this semicircle is given as $\boldsymbol{R}$. We let $\boldsymbol{R} \rightarrow \infty$ so the vertical line portion of the contour will encompass the entire imaginary axis.

\section{A.5 Specific functional forms of each parametric Budyko equation}

By comparing the specific forms of the parametric Budyko equations (i.e. Eq. (4), (5), (6), and (7)) to the general form, Eq.

420 (12), we see that $z=-1$ for all of them. We substitute $z=-1$ into Eq. (A45) to produce,

$y(-1)=\sum_{r=0}^{\infty} \frac{(-1)^{2 r} \Gamma\left(\frac{1+D r}{C}\right)}{r ! C \Gamma\left(\frac{1+D r}{C}+1-r\right)}=\frac{1}{C} \sum_{r=0}^{\infty} \frac{\Gamma\left(\frac{1+D r}{C}\right)}{\Gamma(r+1) \Gamma\left(\frac{1+D r}{C}+1-r\right)}$.

Substitution of the appropriate expressions for $y$ (i.e. $e^{n}$ or $e^{w}$ ), $C$, and $D$ (Table 1) into Eq. (A46) yields the analytical solutions for the two parametric forms of the Budyko equation. Specifically these are:

$n$ for arid climates, $\overline{E_{0}}>\bar{P}$, 
$425 \quad n=\ln \left[\frac{1}{\ln \left(\frac{\bar{E}_{0}}{E}\right)} \sum_{r=0}^{\infty} \frac{\Gamma\left(\frac{1+\ln \left(\frac{\overline{E_{0}}}{P}\right) r}{\ln \left(\frac{E_{0}}{E}\right)}\right)}{\Gamma(r+1) \Gamma\left(\frac{1+\ln \left(\frac{\bar{E}_{0}}{P}\right) r}{\ln \left(\frac{\bar{E}_{0}}{E}\right)}+1-r\right)}\right]$,

$n$ for humid climates, $\overline{E_{0}}<\bar{P}$,

$n=\ln \left[\frac{1}{\ln \left(\frac{\bar{P}}{\bar{E}}\right)} \sum_{r=0}^{\infty} \frac{\Gamma\left(\frac{1+\ln \left(\frac{\bar{P}}{E_{0}}\right) r}{\ln \left(\frac{\bar{P}}{\bar{E}}\right)}\right)}{\Gamma(r+1) \Gamma\left(\frac{1+\ln \left(\frac{\bar{P}}{E_{0}}\right) r}{\ln \left(\frac{\bar{P}}{\bar{E}}\right)}+1-r\right)}\right]$,

$w$ for arid climates, $\overline{E_{0}}>\bar{P}$,

$w=\ln \left[\frac{1}{\ln \left(\frac{E_{0}+\bar{P}-\bar{E}}{\bar{P}}\right)} \sum_{r=0}^{\infty} \frac{\Gamma\left(\frac{1+\ln \left(\frac{\overline{E_{0}}}{\bar{P}}\right) r}{\ln \left(\frac{\bar{E}_{0}+\overline{\bar{P}}-\overline{\bar{E}}}{\bar{P}}\right)}\right)}{\Gamma(r+1) \Gamma\left(\frac{1+\ln \left(\frac{\bar{E}_{0}}{P}\right) r}{\ln \left(\frac{\bar{E}_{0}+\bar{P}-\bar{E}}{\bar{P}}\right)}+1-r\right)}\right]$,

$430 w$ for humid climates, $\overline{E_{0}}<\bar{P}$,

$w=\ln \left[\frac{1}{\ln \left(\frac{E_{0}+\bar{P}-\bar{E}}{\overline{E_{0}}}\right)} \sum_{r=0}^{\infty} \frac{\Gamma\left(\frac{1+\ln \left(\frac{\bar{P}}{E_{0}}\right) r}{\ln \left(\frac{E_{0}+\bar{P}-\bar{E}}{\overline{E_{0}}}\right)}\right)}{\Gamma(r+1) \Gamma\left(\frac{1+\ln \left(\frac{\bar{P}}{E_{0}}\right) r}{\ln \left(\frac{\bar{E} 0_{0}+\bar{P}-\bar{E}}{\overline{E_{0}}}\right)}+1-r\right)}\right]$.

For critical point catchments (i.e. where $\overline{E_{0}}=\bar{P}$ ) the explicit solutions can be found by solving Eq. (4) (or Eq. (6)) and Eq.

(5) (or Eq. (7)) algebraically for $n$ and $w$, respectively, yielding,

$n=\frac{\ln (2)}{\ln \left(\frac{\overline{E_{0}}}{\bar{E}}\right)}=\frac{\ln (2)}{\ln \left(\frac{\bar{P}}{\bar{E}}\right)}$,

435 and

$w=\frac{\ln (2)}{\ln \left(2-\frac{\bar{E}}{\bar{E}_{0}}\right)}=\frac{\ln (2)}{\ln (2-\overline{\bar{E}})}$. 